\title{
Laparoscopic Extirpation of Adrenal Gland Ganglioneuroma Incidentally Diagnosed during Evaluation for Patchy Alopecia Areata in an Adolescent Boy
}

\author{
Zlatan Zvizdic $^{\mathrm{a}}$ Emir Haxhija ${ }^{\mathrm{c}}$ Adisa Chikha $^{\mathrm{b}}$ Emir Milisic $^{\mathrm{a}}$ Asmir Jonuzi $^{\mathrm{a}}$ \\ Semir Vranic ${ }^{d}$ \\ a Department of Pediatric Surgery, University Clinical Center Sarajevo, Sarajevo, Bosnia and Herzegovina; \\ ${ }^{\mathrm{b}}$ Department of Pathology, University Clinical Center Sarajevo, Sarajevo, Bosnia and Herzegovina; \\ 'Department of Pediatric and Adolescent Surgery, Medical University Graz, Graz, Austria; ${ }^{\mathrm{d}}$ College of Medicine, \\ QU Health, Qatar University, Doha, Qatar
}

\section{Significance of the Study}

- The association between alopecia and adrenal gland ganglioneuroma has not been previously reported. Alopecia, in a 17-year-old boy with adrenal ganglioneuroma, resolved following the removal of the tumor and vitamin D supplements. However, alopecia recurred despite normal serum vitamin D levels and no tumor recurrence.

\section{Keywords}

Ganglioneuroma · Adrenal gland · Alopecia areata · Laparoscopy

\begin{abstract}
Objective: We present a 17-year-old boy with an incidentally diagnosed left adrenal ganglioneuroma during the diagnostic workup of alopecia areata. Clinical Presentation and Intervention: Laboratory investigations revealed vitamin D deficiency. Laparoscopic adrenalectomy was performed and ganglioneuroma was confirmed histologically. At follow-up, the vitamin D supplements improved the vitamin $D$ levels followed by a gradual regression of alopecia areata. However, it recurred 18 months later despite the normal levels of serum vitamin D and no tumor recurrence. Con-
\end{abstract}

clusion: Further studies should reveal the relationship between alopecia areata and ganglioneuroma as well as the role of vitamin D in alopecia areata.

(C) 2019 The Author(s)

Published by S. Karger AG, Basel

\section{Introduction}

Ganglioneuroma (GN), a well-differentiated benign neoplasm, belongs to the group of neuroblastic tumors originating from neural crest cells that comprise a spectrum of both benign (e.g., GN) and malignant tumors (e.g., neuroblastoma). The reported incidence of GN is approximately one per million in the general population [1]. GNs occur in all age groups but are more common in older children and young adults, without gender pref-

\begin{tabular}{ll}
\hline KARGER & $\begin{array}{l}\text { (c) } 2019 \text { The Author(s) } \\
\text { Published by S. Karger AG, Basel }\end{array}$ \\
E-Mail karger@karger.com & $\begin{array}{l}\text { This is an Open Access article licensed under the Creative Commons } \\
\text { Attribution-NonCommercial-4.0 International License (CC BY-NC) } \\
\text { (http://www.karger.com/Services/OpenAccessicense), applicable to } \\
\text { the online version of the article only. Usage and distribution for com- } \\
\text { mww.karger.com/mpp }\end{array}$ \\
&
\end{tabular}




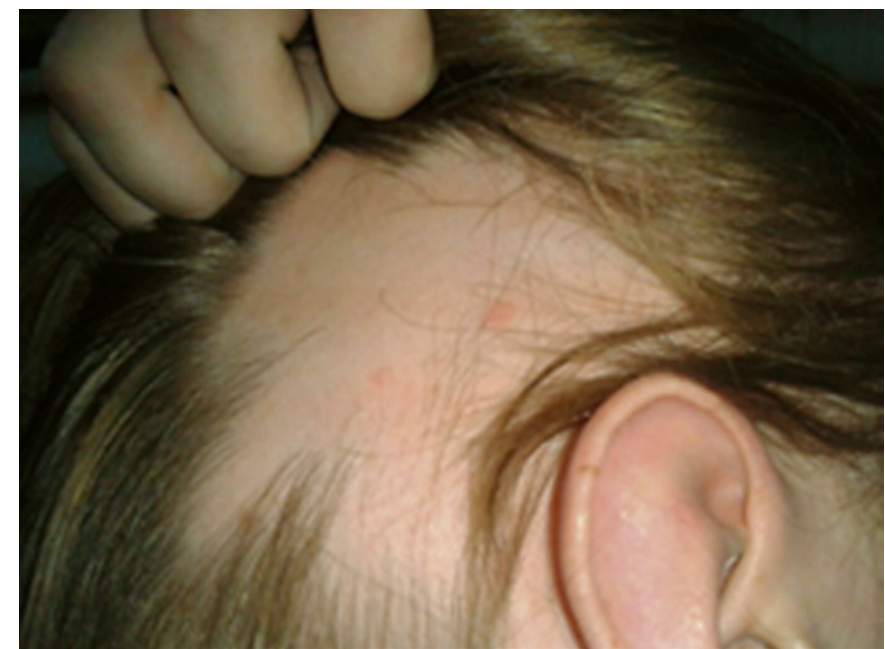

Fig. 1. Clinical presentation of patchy alopecia areata at the nape of the scalp.

erence [2]. Although the adrenal gland is a common site of GN [1], adrenal GNs are rare, constituting $<5 \%$ of all adrenal tumors [3]. Adrenal GNs are often detected incidentally, are hormonally inactive, and are generally without any clinical symptomatology, making their diagnosis difficult. The differential diagnosis of an incidental adrenal mass comprises various primary (benign and malignant) and metastatic tumors. The majority of the incidental adrenal masses ("adrenal incidentalomas") are nonfunctional, benign lesions ( $82.5 \%$ of cases) and among them, the most common ones are adrenal cortical adenomas, myelolipomas, and adrenal cysts, while the rarest ones are GNs [4]. GNs are mostly sporadic but can also be associated with neurofibromatosis type 1 and multiple endocrine neoplasia type 2 syndromes [5]. With the widespread use of radiological studies such as ultrasonography, computed tomography (CT), and magnetic resonance imaging (MRI), the incidental detection of GN has become more common [6]. Although CT and MRI can successfully characterize the nature of the adrenal lesion, these radiological means are insufficient for the final diagnosis of GNs. Due to its rarity and lack of specific radiological findings, the diagnosis is confirmed by postoperative histopathological analysis [6]. Surgery is a preferred mode of treatment for GNs, with an excellent outcome in the vast majority of cases [1].

We present here a rare case of an adrenal GN in a 17 -year-old boy, incidentally diagnosed during the diagnostic assessment for patchy alopecia areata (AA), which also revealed a deficiency in vitamin $\mathrm{D}$. The tumor was successfully treated by laparoscopic adrenalectomy. To the best of our knowledge, this is the first report that describes the coexistence of adrenal GN and AA in a young patient presenting a dilemma of whether it is a true association or a coincidence.

\section{Case Report}

A 17-year-old adolescent boy with a diagnosis of AA at the nape of the scalp (Fig. 1) was referred to the Department of Pediatric Surgery from the primary healthcare unit for further evaluation of the left adrenal mass. The adrenal mass was incidentally detected during the diagnostic workup of AA.

Laboratory investigations for complete blood count, serum electrolytes, immunoglobulins, and urine were normal. Endocrine tests including cortisol, adrenocorticotropic hormone levels, and 24-hour urinary catecholamines were within normal ranges. Routine tumor markers were all negative. Blood pressure was within the normal range. Only vitamin D levels were lower: $12.6 \mathrm{ng} / \mathrm{mL}$ and $27 \mathrm{ng} / \mathrm{mL}$ (measured twice preoperatively).

Physical examination revealed no abnormalities. Ultrasonography of the abdomen revealed a heterogeneous, well-defined mass, measuring $3 \times 2.5 \times 3 \mathrm{~cm}$, located in the topography of the left adrenal gland. An MRI of the abdomen showed a well-defined encapsulated heterogeneously enhancing left adrenal mass, measuring $3 \times 2.8 \mathrm{~cm}$, free from surrounding structures, with a low signal intensity on T1-weighted images and a high signal intensity on T2-weighted images (Fig. 2a). The right adrenal gland was nor$\mathrm{mal}$, and lymphadenopathy was not observed. A diagnosis of nonfunctioning, probably benign, left adrenal tumor was presumed. Due to the absence of precise diagnosis of adrenal lesion after diagnostic workup, an operative treatment was indicated. The patient underwent laparoscopic left adrenalectomy through a transabdominal lateral approach. Four $10-\mathrm{mm}$ trocars were placed in the midclavicular, anterior axillary, midaxillary, and posterior axillary lines. Splenic flexure of transverse colon and spleen were mobilized from their retroperitoneal attachments and incision of retroperitoneum was made along the lateral side of the spleen. After exposure of the adrenal gland, a harmonic scalper was used to dissect the tissue surrounding the gland. By blood vessel dissection, the adrenal gland along with the tumor was removed completely (Fig. 2b). The surgical sample was submitted for histopathologic evaluation. Histomorphologic findings of the mass (Fig. 3a, b; microscopic description provided below the image), consistent with a GN, were further supported by S-100 and CD56-positive tumor cells by immunohistochemistry (Fig. 3c, d).

The postoperative course was uneventful, and the patient was discharged on the fifth day. One-year follow-up consisted of visits every 3 months till the end of the first year after the operation and revealed no recurrence of the tumor. Serum and urine cortisol, adrenocorticotropic hormone, and catecholamines were within normal values. The patient was also treated with vitamin D supplements to correct the vitamin D deficiency. Gradual regrowth of hair at the site of patchy alopecia at 6 months was observed, but AA recurred 18 months later. The treatment with vitamin D supplements improved the serum vitamin $\mathrm{D}$ levels to normal values (last check and follow-up: November 2018). 


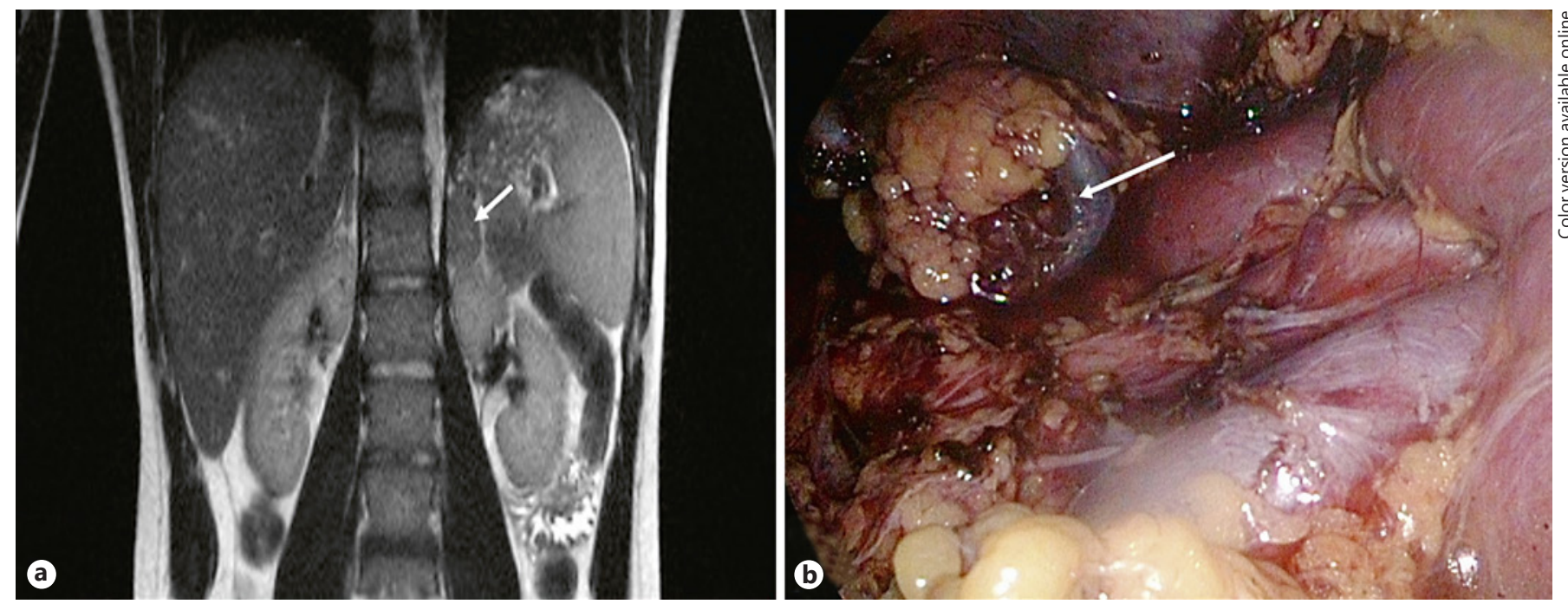

Fig. 2. a Coronal section of MRI T2-weighted image showed a well-defined encapsulated left adrenal mass (white arrow). b Intraoperative image of the left adrenal mass in situ.
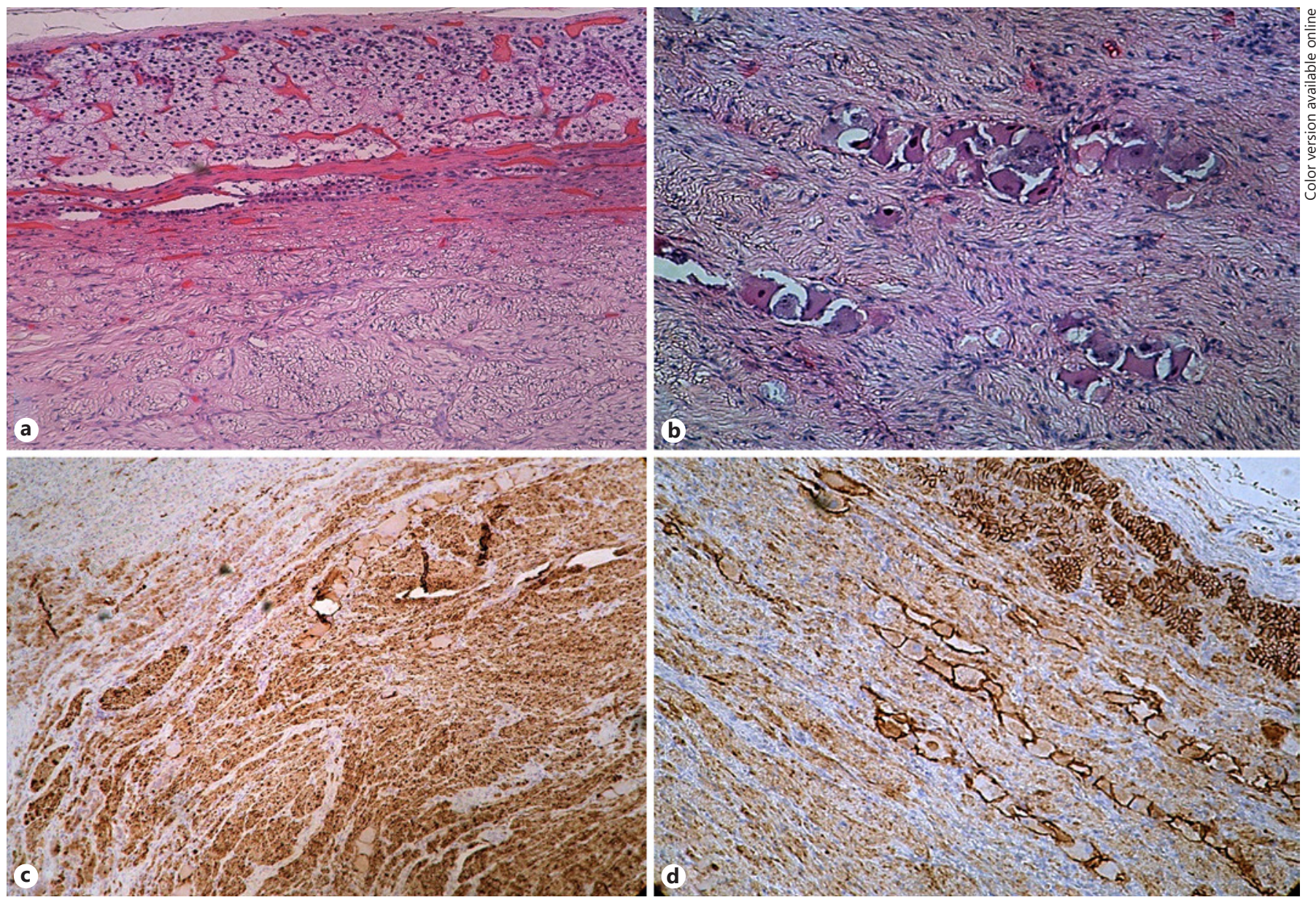

Fig. 3. a, b Hematoxylin and eosin slides showing a well-demarcated, encapsulated mass; note the presence of normal adrenal cortical tissue adjacent to the tumor capsule (upper part of panel $\mathbf{a} ; 10 \times)$. The tumor was composed of mature ganglion cells and Schwann cells, arranged in fascicles; immature cells were absent $(\mathbf{b} ; 20 \times)$. Tumor cells were diffusely and strongly positive for S-100 (c) and CD56 (d; $\times 20)$.

Adrenal Ganglioneuroma in a Patient with Alopecia Areata
Med Princ Pract 2019;28:485-489 DOI: $10.1159 / 000499755$ 
The family history was negative for cancers and autoimmune diseases. No genetic testing or counselling were offered to the patient and his family as these were not available at our hospital.

\section{Discussion}

Although the exact mechanism underlying its pathogenesis is not clear, AA is a T lymphocyte-mediated autoimmune condition that affects genetically susceptible individuals. It is characterized by a patchy nonscarring hair loss, usually on the scalp, eyebrows, eyelashes, beard, or mustache, with a lifetime prevalence of $1-2 \%$ in the general population. Recent studies also revealed an important role for vitamin $\mathrm{D}$ and its receptor in maintaining skin homeostasis including hair follicles [7]. Therefore, vitamin D deficiency has been linked to AA [7]. In our case, the patient had hypovitaminosis $\mathrm{D}$, which improved with vitamin D supplementation. However, a patchy AA recurred despite the treatment and normal serum vitamin D levels, so it remains unclear whether hypovitaminosis D was an underlying mechanism of AA.

An association between GNs and genetic/autoimmune diseases is a topic that has recently been explored. Thus, Lora et al. [8] reported two pediatric cases with multiple endocrine neoplasia type 2 syndromes due to the point mutations in the RET proto-oncogene, and adrenal GNs. The authors concluded that GNs are a rare, but not unexpected, component of the multiple endocrine neoplasia type 2 syndromes. Although much less frequent than pheochromocytoma, GNs have been reported in patients with an autosomal dominant disorder known as neurofibromatosis type 1 or von Recklinghausen disease [8]. In addition, AA has been described as a paraneoplastic phenomenon called primary neoplastic alopecia. Paraneoplastic forms of AA have been described in association with benign and malignant entities arising in the scalp [9]. We have not found any published reports in PubMed/MEDLINE on the potential relationship between GNs or other adrenal tumors (benign or malignant) and AA; thus, the mechanism and potential relationship (if any) remain to be elucidated and require further research at both basic and translational level.

A recent study by Chen et al. [10] analyzed cancer risk in patients with AA. The authors found that three cancer types have elevated risk in AA patients including female breast cancer, kidney and bladder cancer, as well as malignant lymphomas. They concluded that cancer risk in AA patients is organ specific and is not associated with the underlying autoimmune disorders, suggesting that patients with AA and the mentioned cancers share an aberrant molecular activation of the JAK/STAT signaling pathway. The JAK/STAT pathway may be a key molecular driver involved in the pathogenesis of both AA and associated cancers [10]. However, further studies should reveal whether this or some other signaling pathways might be involved in the association between the AA and GN.

Unlike the complete lack of understanding of the possible relationship between GNs and AA, the treatment of GNs in the form of complete surgical excision is well established as the preferred therapeutic modality. Since the early 1990s, laparoscopic adrenalectomy has replaced open adrenalectomy as the gold standard for the surgical removal of most benign adrenal tumors, with a decreased blood loss, shorter hospital stay, shorter convalescence, and diminished patient morbidity in comparison with the open surgery. In contrast, the surgical treatment of adrenal cortical carcinomas is more complex and various factors (e.g., location of the cancer, infiltration of surrounding structures) should be considered when deciding the optimal surgical approach.

\section{Conclusion}

We report for the first time a co-occurrence of an adrenal GN and AA. Alopecia was also associated with vitamin $\mathrm{D}$ deficiency but recurred despite the treatment and normal levels of serum vitamin D. Further studies should explore the relationship between GN and AA, as well as AA and hypovitaminosis D. In addition, we confirm laparoscopic surgery as a safe and effective method for the treatment of adrenal GNs.

\section{Statement of Ethics}

This study has been performed in accordance with the ethical standards from the 1964 Declaration of Helsinki. The study was shared with the local ethical committee, which, however, has the policy not to review case reports.

\section{Disclosure Statement}

The authors declare no conflict of interest. 


\section{References}

1 Spinelli C, Rossi L, Barbetta A, Ugolini C, Strambi S. Incidental ganglioneuromas: a presentation of 14 surgical cases and literature review. J Endocrinol Invest. 2015 May;38(5): $547-54$.

2 Zhou Y, Liang Q, Ou WT, Li ZY, Liu QL. Laparoscopic resection of primary adrenal ganglioneuroma: A case report and review of the literature. Oncol Lett. 2015 May;9(5):216770.

3 Iacobone M, Torresan F, Citton M, Schiavone D, Viel G, Favia G. Adrenal ganglioneuroma: The Padua Endocrine Surgery Unit experience. Int J Surg. 2017 May;41 Suppl 1:S103-8.
4 Baltzer P, Clauser P, Klatte T, Walz J. Workup of the Incidental Adrenal Mass. Eur Urol Focus. $2016 \mathrm{Feb} ; 1(3): 217-22$.

5 De Bernardi B, Gambini C, Haupt R, Granata C, Rizzo A, Conte $\mathrm{M}$, et al. Retrospective study of childhood ganglioneuroma. J Clin Oncol. 2008 Apr;26(10):1710-6.

6 Mylonas KS, Schizas D, Economopoulos KP. Adrenal ganglioneuroma: what you need to know. World J Clin Cases. 2017 Oct;5(10): 373-7.

7 Gerkowicz A, Chyl-Surdacka K, Krasowska D, Chodorowska G. The Role of Vitamin D in Non-Scarring Alopecia. Int J Mol Sci. 2017 Dec;18(12):E2653.
8 Lora MS, Waguespack SG, Moley JF, Walvoord EC. Adrenal ganglioneuromas in children with multiple endocrine neoplasia type 2: a report of two cases. J Clin Endocrinol Metab. 2005 Jul;90(7):4383-7.

9 Geraci AP, de Csepel J, Shlasko E, Wallace SA. Ganglioneuroblastoma and ganglioneuroma in association with neurofibromatosis type I: report of three cases. J Child Neurol. 1998 Jul; 13(7):356-8.

10 Chen CC, Chang YT, Liu HN, Chen YJ. Cancer risk in patients with alopecia areata: a nationwide population-based matched cohort study. Cancer Med. 2018 May;7(5):2153-9. 